\title{
FRAGMENTATION AND THE INITIAL MASS FUNCTION
}

\author{
Richard B. Larson \\ Yale Astronomy Department \\ Box 6666 \\ New Haven, CT 06511, U.S.A.
}

\section{INTRODUCTION}

A central problem in the theory of star formation is to understand the spectrum of masses, or Initial Mass Function, with which stars are formed. The fundamental role of the IMF in galactic evolution has been described by Tinsley (1980), and an extensive review of evidence concerning the IMF and its possible variability has been presented by Scalo (1986). Although the IMF derived from the observations is subject to many uncertainties, two basic features seem reasonably well established. One is that the typical stellar mass, defined such that equal amounts of matter condense into stars above and below this mass, is within a factor of 3 of one solar mass. A theory of star formation should therefore be able to explain why most stars are formed with masses of order one solar mass. The second apparently universal feature is that the IMF for relatively massive stars can be approximated by a power law with a slope not greatly different from that originally proposed by Salpeter (1955). Thus we also need to understand why the IMF always has a similar power-law tail toward higher masses.

Evidence for variability of the IMF has proven slippery, but two possible trends have been suggested by a number of studies. One is that the mass spectra of open clusters may be systematically flatter at high masses than the IMF of field stars, i.e., clusters may contain a higher proportion of the most massive stars (Scalo 1986). A second possible type of variability is that some clusters or regions of star formation may contain relatively few stars with masses below one or two solar masses; this is most strongly suggested for "starburst" regions or systems. The evidence for such variability of the lower IMF, and its possible implications, have been discussed by Larson (1986) and Scalo (1987); however, since it is particularly difficult to establish differences in the lower IMF, we shall focus here on the more well established basic features noted in the previous paragraph.

Circumstantial evidence bearing on the origin of the IMF is provided by the fact that newly formed stars of different masses are typically found in different environments (Herbig 1962; Blaauw 1964; Larson 1982). Low-mass young stars are seen scattered throughout dark clouds such as the nearby Taurus clouds; the fact that they often appear in relative isolation may indicate that they form directly and not by a hierarchical fragmentation process (Herbig 1978). By contrast, massive stars form in a more strongly clustered fashion and only in much larger aggregates of gas and young stars, such as the giant molecular cloud in Orion. The most massive young stars, moreover, are often found in the dense cores of large clusters; examples are the Trapezium system in Orion and the compact 
groups of very massive stars seen at the centers of NGC 3603 and the 30 Doradus nebula (Moffat, Seggewiss, and Shara 1985; Baier, Ladebeck, and Weigelt 1985; Weigelt and Baier 1985). Thus the formation of condensed clusters may play an important role in the formation of the most massive stars.

The observations therefore suggest that the formation of low-mass stars is a primary process involving the fragmentation of clouds into clumps and the collapse of these clumps directly into stars. The formation of massive stars, on the other hand, may be a secondary process that occurs as a result of continuing accumulation or accretion processes in dense environments (Larson 1982). The masses of low-mass stars may then be determined by the mass scale of fragmentation, while the power-law tail of the IMF may be built up through the formation of increasingly massive stars by accretion (Larson 1986). In Sections 4 and 5, it will be suggested that protostellar interactions in a cluster of young stars also play an important role in establishing a mass spectrum, and in Section 6 it will be suggested that feedback effects associated with outflows from young stars can influence its slope.

\section{FRAGMENTATION}

Numerical simulations show that small density fluctuations experience little growth during the early collapse of a nearly uniform cloud, and that the cloud approaches an equilibrium disk, sheet, or filament before fragmentation into clumps becomes marked. This fact, as well as observational evidence, suggests that cloud fragmentation may primarily involve the instability and breakup of nearequilibrium structures such as sheets or filaments (Larson 1985). Detailed studies of the fragmentation of sheetlike clouds by Miyama, Narita, and Hayashi (1987a, b) suggest that sheets tend to fragment into filaments, which then become thinner and finally break into clumps. This type of evolution could account for the observed structure of some star forming clouds that, like the Taurus clouds, consist of clumps strung out along filaments (Schneider and Elmegreen 1979).

The predicted critical mass or "Jeans mass" in the Taurus clouds agrees well with the masses of the dense cores of these clouds, and also with the masses of the newly formed $\mathrm{T}$ Tauri stars associated with them. For a cloud temperature of $8 \mathrm{~K}$, the predicted critical mass is about $1 \mathrm{M}_{\odot}$, which may be compared with a median core mass (measured within the half-maximum $\mathrm{NH}_{3}$ contour) of $0.7 \mathrm{M}_{\odot}$, and a median $\mathrm{T}$ Tauri star mass of $0.6 \mathrm{M}_{\odot}$ (Larson 1985). Thus the dense $\mathrm{NH}_{3}$ cores may indeed have formed by the fragmentation of the filamentary Taurus clouds; once formed, however, these cores apparently collapse with little, if any, further fragmentation to make Tauri stars of comparable mass.

The predicted critical mass depends mainly on the cloud temperature, and is higher in warmer clouds. For example, in the Orion cloud the temperature is typically $\sim 20 \mathrm{~K}$, and the resulting critical mass is a few times larger than in Taurus; this is in qualitative agreement with the fact that both the masses of the molecular clumps and the masses of the known stars are systematically larger in Orion than in Taurus. The median mass of the known T Tauri stars, for example, is about $1.1 \mathrm{M}_{\odot}$ in Orion, compared with $0.6 \mathrm{M}_{\odot}$ in Taurus (Larson 1986). 
It is probably also relevant to cloud fragmentation processes that, while molecular clouds are characterized on larger scales by supersonic turbulent or "non-thermal" motions, the turbulence becomes subsonic on the scale of the cloud cores discussed above (Larson 1981; Myers 1983). In addition, there is evidence that magnetic fields influence the structure and dynamics of larger regions in the Taurus clouds (Heyer et al. 1987) but are relatively unimportant for the cloud cores (Heyer 1988), possibly because the fields have largely decoupled from the cores by ambipolar diffusion (see also Myers and Goodman 1988). These properties suggest that, in addition to the primary role of self-gravity, the dissipation of turbulence and magnetic fields in molecular clouds may also be important in allowing fragmentation and star formation to occur. The development of condensed cloud cores may, in fact, proceed not in a rapid dynamical fashion but in a slow quasi-static fashion modulated by ambipolar diffusion (Shu, Adams, and Lizano 1987; Shu et al. 1988). Nevertheless, a relevant mass scale is still that set by the balance between thermal pressure and gravity, since it is on this scale that nearly spherical and centrally condensed structures capable of collapsing into stars will first form.

\section{FORMATION OF LOW-MASS STARS}

Once a protostellar clump begins to collapse, how does the material in it actually become condensed into a star, and how much of it goes into the star that forms? The formation and growth of a central stellar core or "embryo star" have been extensively studied in the spherical case, and the early stages of this process are probably very similar even when a modest but realistic amount of rotation is present, provided that the collapsing clump is initially centrally condensed (for recent reviews, see Shu, Adams, and Lizano 1987 and Sofia et al. 1989). When rotation is present, the infalling gas eventually begins to accumulate in a disk around the central embryo star, and a substantial fraction of the protostellar material may actually fall into this disk. Considerable evidence suggests that residual disks are in fact common around newly formed stars (e.g. Strom, Edwards, and Strom 1989). It is then of interest to know how much of the material in such a disk is eventually accreted by the central star; clearly this depends on how effectively angular momentum can be transported outward in, or removed from, the disk.

The mechanisms that might transfer angular momentum in protostellar disks have been reviewed by Larson (1989). If the mass of the disk is comparable to or larger than that of the central star, gravitational torques associated with trailing spiral density fluctuations can transfer angular momentum rapidly outward, with the result that at least half of the mass of the star-plus-disk system quickly ends up in the central star. Disk accretion may continue as a result of other effects, which may include the transport of angular momentum by wave motions such as shock waves driven into the disk by tidal interactions with neighboring stars. Another suitable wave source might be a Jupiterlike planet; if such planets form early enough, their perturbing effects may help to disperse the residual gas in protostellar disks, partly by accretion onto the central star, in a time of order $10^{6}$ years that is comparable to the observationally inferred lifetimes of protostellar disks (Larson 1989). 
If most of the gas that falls into a circumstellar disk is eventually accreted by the central star, the final stellar mass is essentially the total mass that falls into the central star and its surrounding disk. Shu et al. (1988) have argued that, since cloud cores are not sharply bounded, infall of gas from the outer parts of the core and from the surrounding cloud would continue almost indefinitely and would build up a star much more massive than a typical $\mathrm{T}$ Tauri star if the infall were not somehow shut off; they suggest that this is accomplished by a stellar wind (see also Shu and Terebey 1984). In this view, it is the onset of a wind associated with the beginning of deuterium burning that is responsible for determining typical stellar masses. However, Stahler (1988) points out that deuterium burning begins undramatically long before a growing embryo star reaches one solar mass, and suggests that it is probably not relevant to determining stellar masses.

The fact that the masses of the T Tauri stars observed in nearby dark clouds are similar to both the predicted "Jeans mass" and the masses of the dense cloud cores suggests, in any case, that cloud properties do play a significant role in determining stellar masses. Even though the cloud cores are not sharply bounded, there is still a central dense region that is predominantly thermally supported, surrounded by a more extended region in which turbulence and magnetic fields provide most of the support. Both turbulence and magnetic fields can inhibit continuing accretion from the region outside the central Jeans-mass core. For example, if the surrounding gas is moving supersonically with respect to the core, the accretion rate predicted by classical accretion theory is reduced by a factor that is approximately the cube of the Mach number (Bondi 1952; Hunt 1971). Magnetic stresses can also prevent the collapse of the outer parts of protostellar clouds and limit the efficiency of star formation (Mouschovias 1987). Stellar winds can further limit the growth of forming stars, either directly by blowing away cloud material (Mathieu et al. 1988) or indirectly by increasing the general level of turbulence in collapsing clouds (see Section 6). Probably a complex interplay of many effects will need to be understood before a fully quantitative theory of stellar masses is possible.

Meanwhile, it may be worth noting that the Jeans mass $M_{\mathrm{J}}$ can be expressed in terms of the sound speed $c$ and the gas pressure $P$ as $M_{\mathrm{J}} \sim c^{4} / G^{3 / 2} P^{1 / 2}$. Although the thermal pressure varies strongly with location in molecular clouds, the total pressure, including turbulent and magnetic contributions, is much more nearly constant everywhere. This follows from the observation that star forming clouds always have similar surface densities, and the fact that the pressure in a selfgravitating cloud is proportional to the square of the surface density (e.g. McKee and Lin 1988). If the supporting pressure is primarily magnetic in origin, then observed cloud properties imply a field strength of the order of $30 \mu \mathrm{G}$ in star forming clouds (Myers and Goodman 1988), but any kind of supporting pressure would be equally compatible with these observations as long as it is nearly constant everywhere. If this universal cloud pressure is combined with a sound speed of $0.2 \mathrm{~km} / \mathrm{s}$ in the above expression for the Jeans mass, then the resulting mass is just under one solar mass. 


\section{DYNAMICS OF PROTOCLUSTERS}

Unlike low-mass stars, massive stars form only in large aggregates of gas and young stars, and the most massive stars seem to form preferentially in the dense cores of massive clusters. This suggests that the dynamics and evolution of clusters of forming stars may play an important or even essential role in the formation of massive stars and in the development of the upper part of the IMF.

Numerical simulations and observations both suggest that the most massive stars form as a result of the continuing accretional growth of a few favored objects (Larson 1978, 1982). Such a process can also generate an upper IMF that is of power-law form (Zinnecker 1982). The tendency of massive stars to form in dense regions might then be a result simply of the fact that accretion proceeds fastest where the density is highest. Another effect that can occur in dense clusters of forming stars is that interactions involving protostellar disks may trigger episodes of enhanced disk accretion (Larson 1982). Interactions can also transfer material between protostellar disks, generally from smaller to larger ones, so that "the rich get richer"; for example, if a star with a disk passes through a denser and more massive disk surrounding a more massive star, its disk may be stripped away and added to the disk around the more massive star. Similar effects occur when galaxies interact: tidal interactions can trigger rapid gas inflows toward the centers of galaxies, and large galaxies can steal material from smaller ones, or even swallow them completely, thereby growing at their expense. In clusters of forming stars, as in clusters of galaxies, the sizes of disks may not be negligible compared to typical encounter distances, so that analogous phenomena may occur.

An example of an environment where interactions may be important is provided by the very dense cluster of young stars around the Trapezium in Orion, which has a density of at least 3000 solar masses per $\mathrm{pc}^{3}$ (Herbig 1983; Herbig and Terndrup 1986). Within a period of $10^{6}$ years, about the present age of this cluster, most of the stars will pass within $1000 \mathrm{AU}$ of another star, and about 10 percent will pass within $100 \mathrm{AU}$ of another star, close enough to strongly disturb a protostellar disk. Encounters would be even more important if the positions or velocities of the stars were initially correlated, for example if they had formed in subclusters (see below).

If a star passes close to or through a disk around another star, it will also experience a gravitational drag that is essentially similar to the "dynamical friction" effect of stellar dynamics. If the star is sufficiently decelerated, which can happen if the disk is massive enough or if the impact parameter of the encounter is small enough, it will be captured into a bound orbit around the other star, forming a binary system. Even when captures do not occur, energy will still be removed from the orbital motions of the stars in a young cluster by this effect, and this will cause the entire cluster to become more condensed. Since the deceleration experienced by a star is proportional to its mass, regardless of whether it interacts with gas or with other stars, the drag effect is strongest for the most massive stars, which therefore become most concentrated at the center of the cluster. The segregation of massive stars toward the center may further accelerate their relative growth, since the residual gas in the cluster will also tend to settle toward the center. Young clusters may in this way become 
dominated by tight central groups of very massive stars, as is indeed observed to be the case in the examples mentioned in Section 1.

If the distribution of matter in a star forming cloud is very clumpy, the stars that eventually make up a cluster may initially form in subclusters that later merge to produce a more massive and centrally condensed system, perhaps with the help of the dynamical friction effect discussed above. Again, such processes may parallel those that occur on galactic scales, since mergers can also build larger galaxies from smaller ones. Subclustering is presently observed in the Trapezium region in the form of a second tight group of massive stars that are now detected only as infrared sources in the core of the molecular cloud, but that will probably soon become visible and join the Trapezium cluster. Subclustering on various scales seems, in fact, to be a nearly universal characteristic of regions of star formation (e.g. Blaauw 1964; Larson 1982; Wilking and Lada 1985).

If the development of larger and more centrally condensed clusters is accompanied by the formation of progressively more massive stars, a power-law IMF might result if the processes involved are self-similar and have no preferred mass scale. The development of a self-similar or "fractal" hierarchy of clustering was suggested by Larson (1978) on the basis of numerical simulations of fragmentation which in some cases showed at least two levels of clustering (see, for example, Fig. 6(a) of that paper). Gravitational drag and accretion effects were also found to be important in these simulations, especially for the most massive objects, which tend to be located at the centers of groups, as expected. Although these simulations are too crude to predict an IMF with any reliability, the slope estimated by counting objects in the two largest mass bins is $x \sim 1.5$, not inconsistent with observed values (cf. Salpeter's value $x=1.35$, where $d N / d \log m \propto m^{-x}$.)

\section{FORMATION OF MASSIVE STARS}

Clearly, we still need to understand in more detail how massive stars form before we can fully understand the upper IMF. The formation of massive stars is less well understood than the formation of low-mass stars, partly because the nearest examples are farther away and more heavily obscured, but it is clear that the formation of massive stars must be a more complex process. One reason is that, unlike the small quiescent cloud cores in which low-mass stars form, molecular clumps that are massive enough to form the most massive stars always contain supersonic internal turbulent motions, and they generally also contain more than one Jeans mass. Therefore they probably evolve in a less regular fashion than smaller clumps and form more than one star, consistent with the evidence that massive stars generally form in groups and clusters (Larson 1981).

Another difference is that for massive stars, radiation pressure eventually becomes important during the star formation process and prevents further gas infall from occurring, at least in the case of spherical collapse. Wolfire and Cassinelli (1987) find, in fact, that radiation pressure is even more important than was estimated by Larson and Starrfield (1971) and Kahn (1974), and prevents accretion from continuing for a star more massive than about $15-30 \mathrm{M}_{\odot}$. Radiation pressure can be overcome by non-spherical accretion from a dense sheet or disk of gas (Nakano 1989), but all of the 
matter destined to make a massive star must in this case condense into such a flattened structure before more than a small fraction of it has gone into a star. Another possible source of already condensed gas from which to make a massive star might be circumstellar disks around smaller stars, which are swept up and added to the disk around a massive star. If massive stars form in close multiple systems, actual stellar collisions and mergers might also occur, and might provide an especially effective way of building up very massive stars without hindrance from radiation pressure.

A final difference from low-mass stars is that the formation of massive stars almost certainly takes longer than the formation of low-mass stars, simply because the building materials must be assembled from a larger region that has a longer dynamical timescale. All of these differences reinforce the general conclusion that stars of larger mass form at later stages in the development of progressively more condensed aggregates of gas and stars. However, the actual processes involved remain unclear, and could include accretion from massive and dense disks, theft of additional disk material from smaller stars, and even stellar cannibalism.

A common feature of most such scenarios is that massive stars form from residual material left over from the prior formation of large numbers of less massive stars. A general implication of this is that most of the matter that condenses into stars will go into low-mass stars, and a decreasing amount will remain to make progressively more massive stars. For a power-law IMF, this implies that $x>1$. For example, the Salpeter slope $x=1.35$ could plausibly be produced in this way; if stars of larger mass form at later times, and if the amount of gas condensing into new stars decreases by 20 percent with each factor of 2 increase in the maximum stellar mass, then a mass spectrum with $x=1.32$ is produced.

If clustering plays an important role in the formation of massive stars, another expectation would be that cluster mass spectra should contain a higher proportion of massive stars than the IMF of field stars. As noted earlier, there is marginal evidence for such a difference; the studies reviewed by Scalo (1986) yield $x \sim 1.2 \pm 0.5$ for open clusters, compared with $x \sim 1.7 \pm 0.5$ for field stars when the data are analyzed in the same way. There is also some evidence that the upper IMF is flatter in large clusters than in small ones (Burki 1977). Very flat mass spectra with $x \sim 0.5$ have been found by Elson, Fall, and Freeman (1989) for several Large Magellanic Cloud clusters, although in apparent conflict with this result, Mateo (1988) finds steeper spectra with $x>2$ for a number of other Magellanic Cloud clusters. Clarification of this presently confused subject would clearly be desirable, and could yield fundamental clues regarding the origin of the IMF.

\section{FEEDBACK AND THE IMF}

It has often been suggested that negative feedback effects regulate the efficiency and the rate of star formation in galaxies by destroying star forming clouds or by supporting them against collapse (see, for example, Larson 1987). Self-regulation effects can also influence the IMF, especially if the more massive stars generate stronger feedback effects and if this limits the amount of matter that can condense into massive stars. 
Star forming clouds may eventually be completely destroyed by ionization and strong winds from very massive stars, but even before this happens, bipolar outflows from less massive stars can stir up such clouds and increase the amount of turbulence present in them (Lada 1988). This will provide additional cloud support and may inhibit the continuing growth of forming stars. Evidence that star formation generates turbulence in molecular clouds is provided by the fact that clumps containing young stars generally have larger internal turbulent motions than clumps without stars (Fuller and Myers 1987; Benson and Myers 1989; Loren 1989). A detailed feedback cycle whereby outflows may regulate both star formation and cloud properties has been suggested by McKee and Lin (1988); they argue that outflows will tend to expand a star forming cloud and thus regulate its opacity and degree of ionization so as to keep the rate of star formation, which is presumed to be controlled by ambipolar diffusion, just sufficient to resupply the mechanical energy being dissipated in the cloud. Such a mechanism could account not only for the nearly constant surface density of starforming clouds, but also for the typical low efficiency of star formation.

If stars of larger mass form at later stages in the development of more condensed aggregates, the amount of matter going into stars of each mass may also be regulated by feedback effects like those discussed above. If the mechanical energy deposited in the cloud per unit mass of stars formed is an increasing function of stellar mass, this could have the consequence that progressively smaller amounts of matter condense into stars of increasing mass. This might provide a more physical basis for the scheme suggested in Section 5 to produce a power-law IMF with $x>1$, where a decreasing amount of residual matter was assumed to condense into stars of increasing mass.

To illustrate by a highly oversimplified example how feedback might control the slope of the IMF, suppose that a constant fraction (of order $10^{-3}$ ) of the binding energy of each star is converted via outflows into mechanical energy that contributes to the support of the cloud. If the most massive star in each clump or subcluster grows in mass until the total mechanical energy generated by it is equal to the binding energy of the clump, the binding energy of the most massive star is then a constant multiple (of order $10^{3}$ ) of the clump binding energy. Assuming that the radii of accreting stars are approximately independent of mass (Larson 1972; Stahler 1988) and that the binding energies of molecular clumps vary with the 1.4 power of clump mass (Larson 1981), we then predict that the maximum stellar mass increases with the 0.7 power of the clump mass. If aggregates of gas and young stars are built up in such a way that the mass of the most massive star always remains proportional to the 0.7 power of the total mass of gas and stars present, and if a nearly constant fraction of this total mass is in stars, then a power-law IMF is built up whose slope is $x=1 / 0.7$, or $x=1.4$.

Of course, this example neglects many complications, and can hardly be considered a convincing prediction of the IMF; for example, it is not fully consistent with the conclusion of Section 5 that the most massive stars form only from material that is already very condensed and presumably is little affected by feedback effects. Clearly, the full problem of understanding the IMF is exceedingly complex, and all we can really hope for at present is to gain an inkling of some of the many effects involved. In such a situation, the power of theory is limited and the need for 
observational input is great; therefore it is to be hoped that the increasingly detailed data now becoming available on regions of star formation can help to clarify which processes are most important in determining the IMF.

\section{REFERENCES}

Baier, G., Ladebeck, R., and Weigelt, G., 1985. Astr. Astrophys., 151, 61.

Benson, P. J., and Myers, P. C., 1989. Astrophys. J. Suppl., in press.

Blaauw, A., 1964. Ann. Rev. Astr. Astrophys., 2, 213.

Bondi, H., 1952. Mon. Not. Roy. Astr. Soc., 112, 195.

Burki, G., 1977. Astr. Astrophys., 57, 135.

Elson, R. A. W., Fall, S. M., and Freeman, K. C., 1989. Astrophys. J., 336, 734.

Fuller, G. A., and Myers, P. C., 1987. In Physical Processes in Interstellar Clouds, eds. G. E. Morfill and M. Scholer, p. 137. Reidel, Dordrecht.

Herbig, G. H., 1962. Advances Astr. Astrophys., 1, 47.

Herbig, G. H., 1978. In The Origin of the Solar System, ed. S. F. Dermott, p. 219. Wiley, New York.

Herbig, G. H., 1983. Highlights of Astronomy, 6, 15.

Herbig, G. H., and Terndrup, D. M., 1986. Astrophys. J., 307, 609.

Heyer, M. H., 1988. Astrophys. J., 324, 311.

Heyer, M. H., Vrba, F. J., Snell, R. L., Schloerb, F. P., Strom, S. E., Goldsmith, P. F., and Strom, K. M., 1987. Astrophys. J., 321, 855.

Hunt, R., 1971. Mon. Not. Roy. Astr. Soc., 154, 141.

Kahn, F. D., 1974. Astr. Astrophys., 37, 149.

Lada, C. J., 1988. In Galactic and Extragalactic Star Formation, eds. R. E. Pudritz and M. Fich, p.

5. Kluwer, Dordrecht.

Larson, R. B., 1972. Mon. Not. Roy. Astr. Soc, 157, 121.

Larson, R. B., 1978. Mon. Not. Roy. Astr. Soc., 184, 69.

Larson, R. B., 1981. Mon. Not. Roy. Astr. Soc., 194, 809.

Larson, R. B., 1982. Mon. Not. Roy. Astr. Soc, 200, 159.

Larson, R. B., 1985. Mon. Not. Roy. Astr. Soc., 214, 379.

Larson, R. B., 1986. In Stellar Populations, eds. C. A. Norman, A. Renzini, and M. Tosi, p. 101. Cambridge Univ. Press, Cambridge.

Larson, R. B., 1987. In Starbursts and Galaxy Evolution, eds. T. X. Thuan, T. Montmerle, and J. Tran Thanh Van, p. 467. Editions Frontières, Gif sur Yvette.

Larson, R. B., 1989. In The Formation and Evolution of Planetary Systems, eds. H. A. Weaver and L. Danly, in press. Cambridge Univ. Press, Cambridge.

Larson, R. B., and Starrfield, S., 1971. Astr. Astrophys., 13, 190. 
Loren, R. B., 1989. Astrophys. J., 338, 925.

Mateo, M., 1988. Astrophys. J., 331, 261.

Mathieu, R. D., Benson, P. J., Fuller, G. A., Myers, P. C., and Schild, R. E., 1988. Astrophys. $J ., 330,385$.

McKee, C. F., and Lin, J.-Y., 1988. In Origin, Structure, and Evolution of Galaxies, ed. Fang Li Zhi, p. 47. World Scientific, Singapore.

Miyama, S. M., Narita, S., and Hayashi, C., 1987a. Prog. Theor. Phys., 78, 1051.

Miyama, S. M., Narita, S., and Hayashi, C., 1987b. Prog. Theor. Phys., 78, 1273.

Moffat, A. F. J., Seggewiss, W., and Shara, M. M., 1985. Astrophys. J., 295, 109.

Mouschovias, T. Ch., 1987. In Physical Processes in Interstellar Clouds, eds. G. E. Morfill and M. Scholer, p. 453. Reidel, Dordrecht.

Myers, P. C., 1983. Astrophys. J., 270, 105.

Myers, P. C., and Goodman, A. A., 1988. Astrophys. J., 329, 392.

Nakano, T., 1989. Astrophys. J., 344, in press.

Salpeter, E. E., 1955. Astrophys. J., 121, 161.

Scalo, J. M., 1986. Fundamentals of Cosmic Physics, 11, 1.

Scalo, J. M., 1987. In Starbursts and Galaxy Evolution, eds. T. X. Thuan, T. Montmerle, and J. Tran Thanh Van, p. 445. Editions Frontières, Gif sur Yvette.

Schneider, S., and Elmegreen, B. G., 1979. Astrophys. J. Suppl., 41, 87.

Shu, F. H., and Terebey, S., 1984. In Cool Stars, Stellar Systems, and the Sun, eds. S. L. Baliunas and L. Hartmann, p. 78. Springer-Verlag, Berlin.

Shu, F. H., Adams, F. C., and Lizano, S., 1987. Ann. Rev. Astr. Astrophys., 25, 23.

Shu, F. H., Lizano, S., Adams, F. C., and Ruden, S. P., 1988. In Formation and Evolution of Low Mass Stars, eds. A. K. Dupree and M. T. V. T. Lago, p. 123. Kluwer, Dordrecht.

Sofia, S., Kawaler, S., Larson, R., and Pinsonneault, M., 1989. In Solar Interior and Atmosphere, eds. A. N. Cox and M. S. Matthews, in press. Univ. of Arizona Press, Tucson.

Stahler, S. W., 1988. Astrophys. J., 332, 804.

Strom, S. E., Edwards, S., and Strom, K. M., 1989. In The Formation and Evolution of Planetary Systems, eds. H. A. Weaver and L. Danly, in press. Cambridge Univ. Press, Cambridge.

Tinsley, B. M., 1980. Fundamentals of Cosmic Physics, 5, 287.

Weigelt, G., and Baier, G., 1985. Astr. Astrophys., 150, L18.

Wilking, B. A., and Lada, C. J., 1985. In Protostars and Planets II, eds. D. C. Black and M. S. Matthews, p. 297. Univ. of Arizona Press, Tucson.

Wolfire, M. G., and Cassinelli, J. P., 1987. Astrophys. J., 319, 850.

Zinnecker, H. C., 1982. In Symposium on the Orion Nebula to Honor Henry Draper, eds. A. E. Glassgold, P. J. Huggins, and E. L. Schucking, p. 226. Ann. New York Acad. Sci, Vol. 395. 


\section{Discussion:}

OSTERBROCK: Is the estimate that the star density in the 30 Doradus region is $10^{2}$ the density in the Orion Nebula cluster based on the observed numbers of most luminous stars only, or on observed numbers of less luminous stars as well?

LARSON: It is based only on the most luminous stars, with an assumption that a "standard" IMF applies. However, even for the most luminous stars alone, the density in the 30 Doradus core is much higher than in the Trapezium region.

HEILES: In a forming star cloud processes such as solar flares produce enough cosmic rays to cause enough ionization to freeze the magnetic field into the gas?

LARSON: I am not aware that this possibility has been studied carefully. Such effects might well be important close to the central star. However, cosmic rays may not penetrate far into a typical protostellar disk, which is optically highly opaque.

SOLOMON: In one of your scenarios you said that high mass stars form after the low mass stars from "leftover" gas implying high mass star formation was limited by the quantity of available gas. However molecular clouds which are gravitationally bound have $10^{4}, 10^{5}$ or even $10^{6}$ solar masses of material available to form stars. There is NO shortage of gas.

LARSON: If massive stars form in dense clusters in the cores of molecular clouds, the amount of gas in the region where a cluster forms is much less than the total mass. This gas may become strongly depleted by star formation, leaving less to make the most massive stars. However, regardless of whether gas depletion is important, I still think it is likely that the more massive stars form at later stages in the evolution of star forming regions.

KHAN: You did not mention the importance of magnetic fields in the transport of angular momentum. Is this the currently accepted view?

LARSON: It was estimated by Hayashi that throughout most of a protostellar disk, field decay by Ohmic dissipation is very rapid, and this implies that magnetic fields cannot be dynamically important. This conclusion may not apply to the outermost part of a disk, or to the part close to the central star. The outermost part (beyond $100 \mathrm{AU}$ ) could retain some magnetic coupling to the interstellar medium, and the innermost part could be magnetically coupled to the central star, but other mechanisms appear necessary to transport angular momentum in between. 


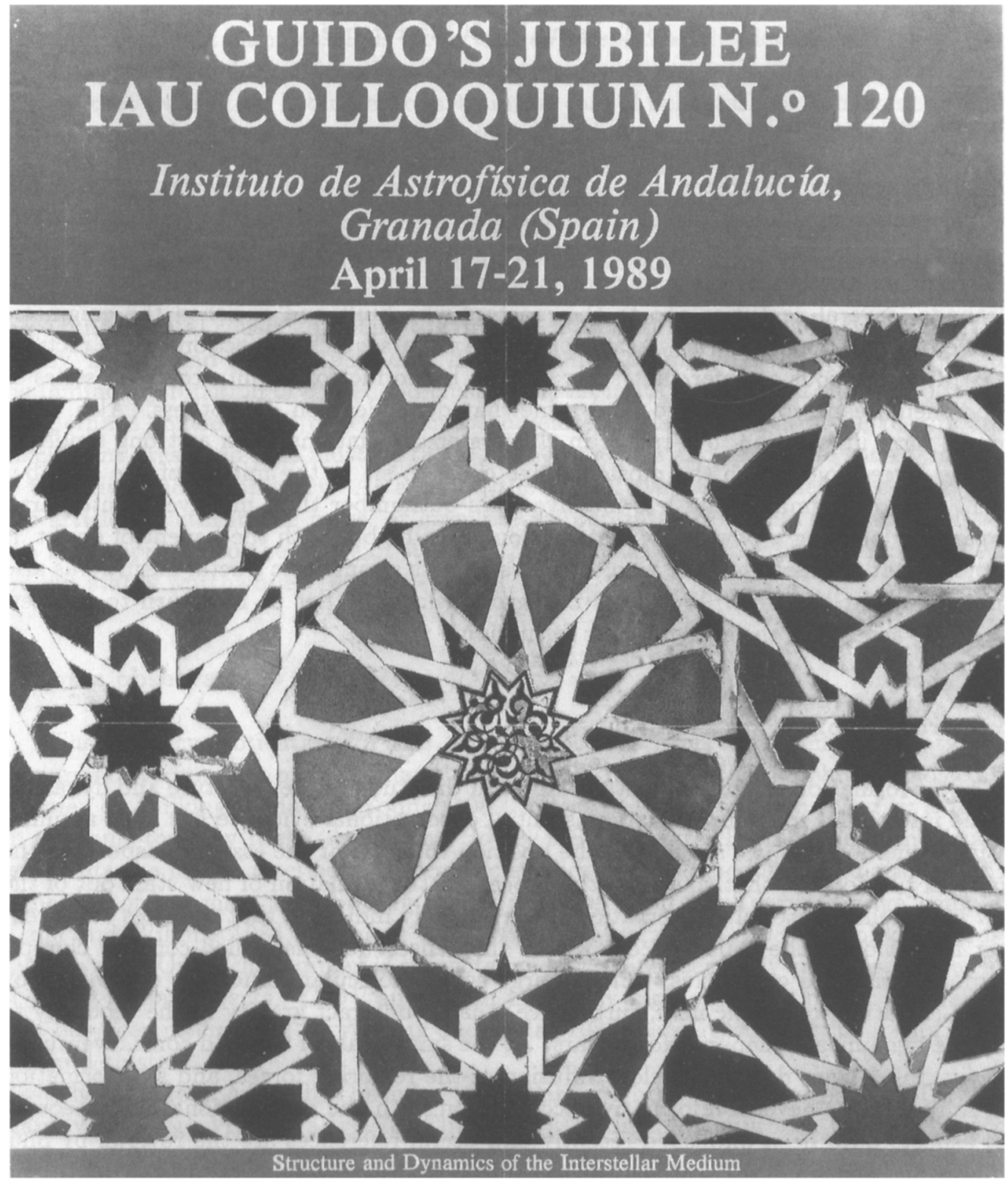

